\title{
Bigenic mouse models of focal segmental glomerulosclerosis involving pairwise interaction of CD2AP, Fyn, and synaptopodin
}

Tobias B. Huber, ${ }^{1}$ Christopher Kwoh, ${ }^{2}$ Hui Wu, ${ }^{1}$ Katsuhiko Asanuma, ${ }^{3}$ Markus Gödel, ${ }^{1}$ Björn Hartleben, ${ }^{1}$ Ken J. Blumer, ${ }^{4}$ Jeffrey H. Miner, ${ }^{2}$ Peter Mundel, ${ }^{3}$ and Andrey S. Shaw ${ }^{1}$

1Department of Pathology and Immunology and 'Department of Medicine, Renal Division, Washington University School of Medicine, St. Louis, Missouri, USA. ${ }^{3}$ Department of Medicine, Mount Sinai School of Medicine, New York, New York, USA. 4Department of Cell Biology and Physiology,

Washington University School of Medicine, St. Louis, Missouri, USA.

Focal segmental glomerulosclerosis (FSGS) is the most common primary glomerular diagnosis resulting in end-stage renal disease. Defects in several podocyte proteins have been implicated in the etiology of FSGS, including podocin, $\alpha$-actinin-4, CD2-associated protein (CD2AP), and TRPC6. Despite our growing understanding of genes involved in the pathogenesis of focal segmental sclerosis, the vast majority of patients with this disease, even those with a familial linkage, lack a clear genetic diagnosis. Here, we tested whether combinations of genetic heterozygosity (bigenic heterozygosity) that alone do not result in clinical kidney disease could function together to enhance susceptibility to glomerular damage and FSGS. Combinations of Cd2ap heterozygosity and heterozygosity of either synaptopodin (Synpo) or Fyn proto-oncogene (Fyn) but not kin of IRRE like 1 (Neph1) resulted in spontaneous proteinuria and in FSGS-like glomerular damage. These genetic interactions were also reflected at a functional level, as we found that CD2AP associates with Fyn and Synpo but not with Neph1. This demonstrates that bigenic heterozygosity can lead to FSGS and suggests that combined mutations in 2 or multiple podocyte genes may be a common etiology for glomerular disease.

\section{Introduction}

Focal segmental glomerulosclerosis (FSGS) is a major cause of endstage renal disease and is increasing in frequency (1). The structure affected in FSGS is the kidney glomerulus, which is composed of capillaries that are involved in the filtration of blood. The diagnosis of FSGS is based on the clinical findings of proteinuria and specific histopathological changes, with areas of glomerular sclerosis and glomerular tuft collapse that are both focal and segmental. Focal refers to the finding that not all glomeruli are affected, and segmental indicates that only a portion of the affected glomerulus is sclerosed. While the etiology of most cases of FSGS is unknown, the heterogeneous nature of these histologic changes suggests that a combination of different factors is involved, including genetic and environmental influences $(2,3)$.

The podocyte, the visceral epithelial cell of the glomerulus, has taken center stage in research on the pathogenesis of FSGS. Genetic studies in both human and mouse reveal that the development of FSGS is initiated by podocyte dysfunction (2). In humans, mutations of the podocyte-specific gene nephrosis 2 homolog, podocin (Nphs2) (encoding podocin) (4), the more ubiquitously expressed cytoskeletal protein $\alpha$-actinin-4 (5), and the ion channel protein TRPC6 $(6,7)$ have been shown to impair podocyte function and to cause an inherited form of FSGS. Mouse studies demonstrate that the complete absence of either podocin (8) or $\alpha$-actinin-4 (9) results not in FSGS but in a severe podocyte dysfunction leading

Nonstandard abbreviations used: ARF, ADP-ribosylation factor; BUN, blood urea nitrogen; CD2AP, CD2-associated protein; FSGS, focal segmental glomerulosclerosis; Fyn, Fyn proto-oncogene; MAP, mean arterial pressure; Neph1, kin of IRRE like 1; Nphs2, nephrosis 2 homolog, podocin; Synpo, synaptopodin.

Conflict of interest: The authors have declared that no conflict of interest exists. Citation for this article: J. Clin. Invest. 116:1337-1345 (2006). doi:10.1172/JCI27400. to a lethal nephrotic syndrome that occurs at or near birth. The complete absence of other gene products that are either podocyte specific, such as nephrin (10) and kin of IRRE like 1 (Neph1) (11), or more ubiquitously expressed, such as CD2-associated protein (CD2AP) (12), can also lead to severe podocyte dysfunction and congenital nephrotic syndrome.

These studies suggest that the degree of podocyte dysfunction determines whether congenital nephrotic syndrome or FSGS occurs. Severe impairment of podocyte function would lead to perinatal glomerular failure while a more modest impairment would require additional environmental factors before focal and segmental changes in glomeruli could occur (2).

In this study, we were interested to explore whether combinations of heterozygosity for different podocyte-relevant genes would increase the risk for FSGS. We therefore crossed mice heterozygous for $C d 2 a p$ with mice that are heterozygous for the signaling protein Fyn proto-oncogene (Fyn), the long isoform of the actin-bundling protein synaptopodin (Synpo), or the slit diaphragm protein Neph1. While Cd2ap-null mice develop nephrotic syndrome and end-stage renal disease early in life, the clinical phenotype of $C d 2 a p$ heterozygous animals is relatively unremarkable (13). However, bigenic heterozygosity of Cd2ap with Fyn or Synpo but not Neph1 resulted in a significantly increased incidence of proteinuria and pathological changes consistent with FSGS. This suggests that combined mutations of genes in pathways involving CD2AP, Fyn, and Synpo may account for a significant fraction of patients with FSGS.

\section{Results}

Bigenic heterozygosity of $C d 2$ ap and Fyn leads to proteinuria. We first bred Cd2ap heterozygous mice to Fyn-deficient mice on a pure C57BL/6J background. Fyn is a Src-family tyrosine kinase that is 
A

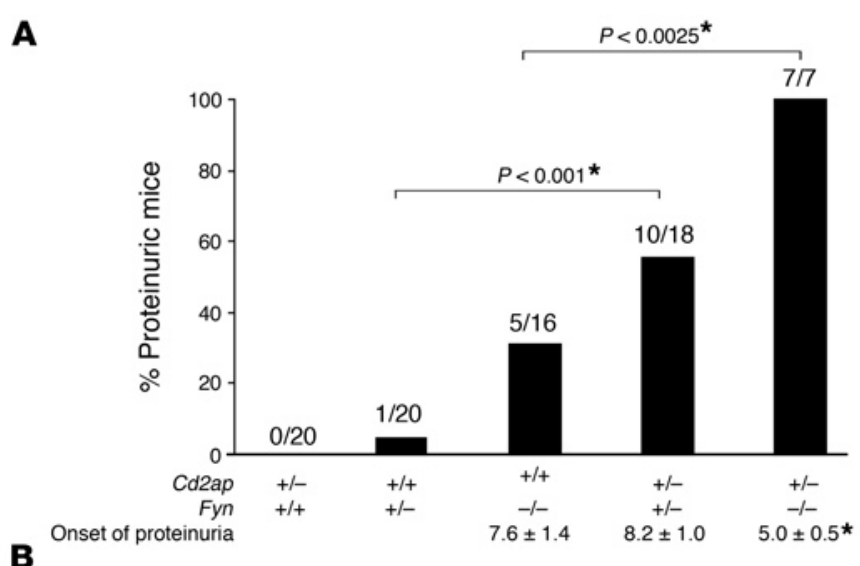

B

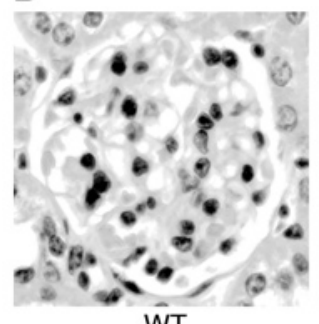

WT

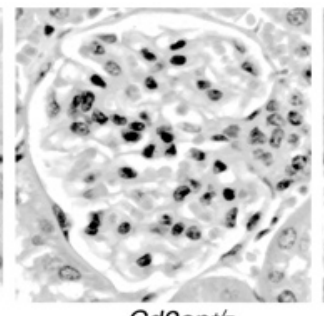
Cd2ap $+1-$

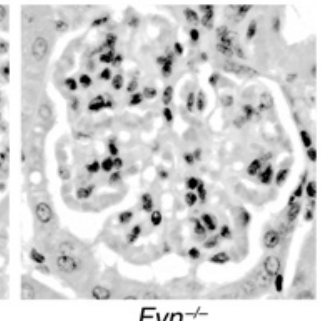
Fyn $^{-1-}$
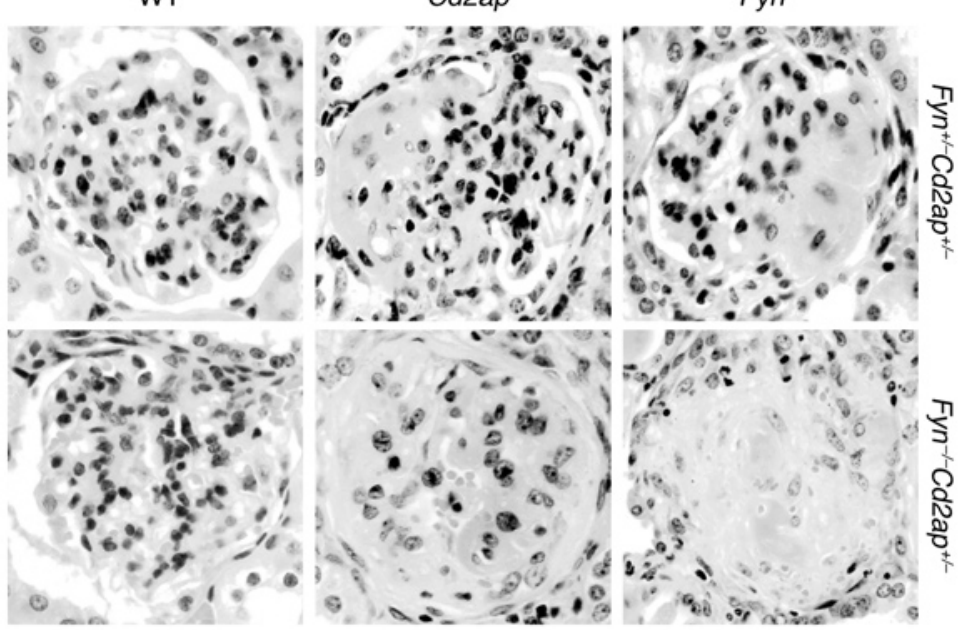

Figure 1

CD2AP haploinsufficiency enhances proteinuria in Fyn-deficient mice. (A) Mice were tested monthly for proteinuria. The incidence of proteinuria is depicted as both percentages and absolute numbers for the indicated mouse genotypes followed to up to 12 months of age. The onset of proteinuria is shown below for each genotype in months \pm SEM. *Statistical significance. (B) Sections of formalin-fixed kidney cortex from 5- to 8-month-old mice were stained with H\&E and with PAS. In $\mathrm{Fyn}^{-1-}$ mice, most glomeruli were normal (top right). Representative glomeruli from $\mathrm{Fyn}^{+/-} \mathrm{Cd} 2 \mathrm{ap}^{+/-}$mice (middle row) or $\mathrm{Fyn}^{-1-} \mathrm{Cd} 2 \mathrm{ap}^{+--}$mice (bottom row) taken 2 months after the onset of proteinuria reveal mesangial hypercellularity and segmental sclerosis or global sclerosis in some glomeruli. Magnification, $\times 400$.

within 1 month of detection. In our colony, about 31\% of $\mathrm{Fyn}^{-/-}$mice developed proteinuria, with an average age of onset of 8 months of age, a phenotype that is intermediate between those in the 2 previous studies. Consistent with what was reported previously, $\mathrm{Fyn}^{+/-}$mice did not develop proteinuria $(14,15)$.

In the presence of CD2AP haploinsufficiency, $56 \%$ of $\mathrm{Fyn}^{+/-}$mice developed proteinuria $(P<0.01$ relative to $\mathrm{Fyn}^{+/-}$alone) while the rate of proteinuria in $\mathrm{Fyn}^{-/-}$mice was increased to $100 \%$ (Figure $1 \mathrm{~A} ; P<0.0025$ ). In addition, CD2AP haploinsufficiency significantly accelerated the onset of proteinuria from 8 months of age to 5 months of age $(P<0.05)$. Near the onset of proteinuria, the serum blood urea nitrogen (BUN) $(16.6 \pm 1.3 \mathrm{mg} / \mathrm{dl})$

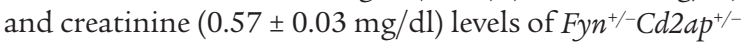
and $\mathrm{Fynn}^{-/-} \mathrm{Cd} 2 \mathrm{ap}^{+/-}$mice were similar to those of wild-type mice (C57BL/6 background). Five mice were measured 2-3 months after onset of proteinuria, with consistently elevated creatinine levels $(0.7 \pm 0.03 \mathrm{mg} / \mathrm{dl})$ and slightly elevated BUN levels $(18.8 \pm 3.1 \mathrm{mg} / \mathrm{dl})$. Proteinuria progressed in these mice from $70 \pm 12 \mathrm{mg} / \mathrm{dl}$ to $200 \pm 41 \mathrm{mg} / \mathrm{dl}$ $(n=5)$ over this period of time.

Light microscopic examination of Fyn ${ }^{-/+}$and Fyn ${ }^{-/-}$were consistent with previous studies and showed no significant or only minor histological changes, such as mesangial hypercellularity in some Fyn ${ }^{-/}$glomeruli. However, in

expressed in podocytes, where it has been shown to phosphorylate the slit diaphragm component nephrin (14). While Yu et al. reported that most $\mathrm{Fyn}^{-1-}$ mice develop mild proteinuria by 6 months of age, Verma et al. were unable to detect any significant proteinuria in $\mathrm{Fyn}^{-1-}$ mice $(14,15)$. Both groups found no histological abnormalities but did demonstrate podocyte foot process effacement. Lastly, $\mathrm{Fyn}^{+-}$mice do not develop proteinuria or display any light or electron microscopic abnormalities (15).

Five mouse genotypes were generated and followed up to 12 months: $\mathrm{Fyn}^{+/+} \mathrm{Cd} 2 \mathrm{ap}^{+/-}, \mathrm{Fyn}^{+/-} \mathrm{Cd} 2 \mathrm{ap}^{+/+}, \mathrm{Fyn}^{-/-} \mathrm{Cd} 2 \mathrm{ap}^{+/+}, \mathrm{Fyn}^{+/-}$ $\mathrm{Cd} 2 \mathrm{ap}^{+/-}$, and $\mathrm{Fyn}^{-1-} \mathrm{Cd} 2 \mathrm{a} \mathrm{p}^{+-}$. Because proteinuria is the earliest and most sensitive clinical indicator of glomerular dysfunction, proteinuria was used as the primary end-point for this study. Mice were tested monthly for the presence of albumin in the urine and followed to up to 12 months of age. Because some wild-type mice develop low-grade proteinuria at advanced ages, varying with genetic background (16-18), we used only littermates for controls. Proteinuric animals were typically sacrificed the presence of CD2AP haploinsufficiency, there were significant pathological glomerular changes, some of which were consistent with FSGS. The most common finding was glomerular hypercellularity with mesangial expansion seen in many but not all glomeruli (Figure 1B). There were occasional sclerotic glomeruli and glomeruli with segmental sclerosis (Figure 1B). There was a tendency toward more significant levels of sclerosis in the $\mathrm{Fyn}^{-/-} \mathrm{Cd} 2 \mathrm{ap}{ }^{+/}$ mice compared with $\mathrm{Fyn}^{+/-} \mathrm{Cd} 2 \mathrm{ap}^{+/-}$mice. The histological changes correlated with the degree of proteinuria.

Electron microscopic examination of $\mathrm{Fyn}^{+/-}$and $\mathrm{Fyn}^{-/-}$kidneys demonstrated mild changes, with foot process coarsening, fusion, and effacement consistent with previous reports. In the presence of CD2AP haploinsufficiency, these changes were more extensive (Figure 2). In proteinuric animals, electron-dense subepithelial and mesangial deposits were detected (Figure 2).

Bigenic heterozygosity of Cd2ap and Synpo leads to proteinuria. We next bred Cd2ap heterozygous mice to Synpo-deficient mice on a pure 129 background. Synpo is an actin-bundling protein 
A

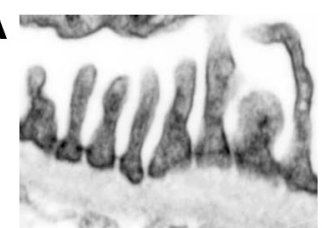

WT

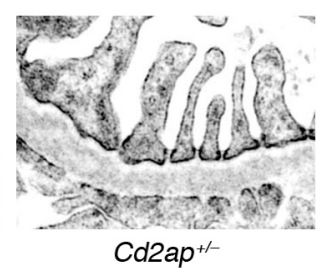

Cd2apt-1

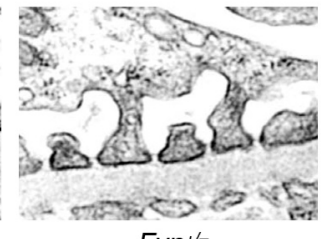

$\mathrm{Fyn}^{+1-}$
B

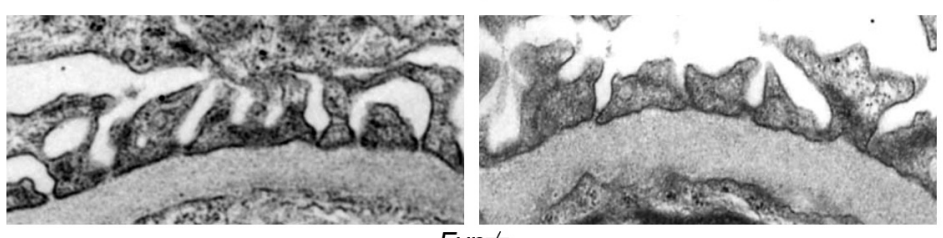

Fyn $^{-1-}$
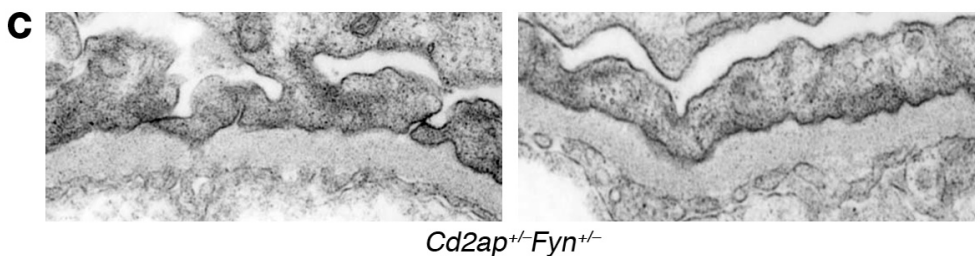

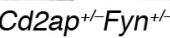

D
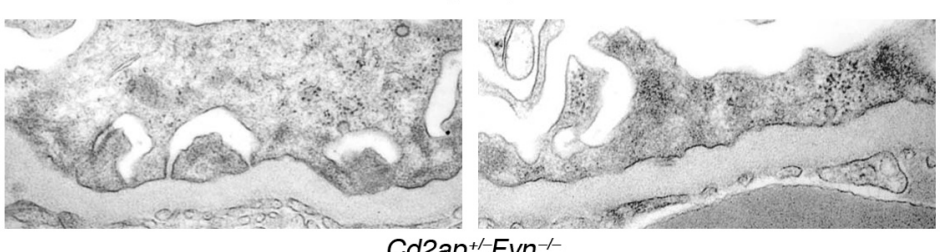

Cd2ap ${ }^{+/-} \mathrm{Fyn}^{-1}$

expressed specifically in podocytes and in telencephalic dendrites (19). There are 3 isoforms of Synpo (Synpo-long, Synpo-short, and Synpo-T) that are generated by alternative RNA splicing (20). Recently generated Synpo-/- mice lack the Synpo-short and Synpo-long expression but still express Synpo-T isoform in podocytes (20). While these mice exhibit neuronal defects, no spontaneous kidney phenotype was detected $(20,21)$.

Four genotypes were generated: Synpo ${ }^{+/+} \mathrm{Cd}_{2} \mathrm{ap}^{+/}$, Synpo $\mathrm{s}^{-/}$ $\mathrm{Cd} 2 \mathrm{ap}^{+/+}$, Synpo $^{+/-} \mathrm{Cd} 2 \mathrm{ap}^{+/-}$, and Synpo ${ }^{-/-} \mathrm{Cd} 2 \mathrm{ap}^{+/-}$. The animals were evaluated in the same manner as the $\mathrm{Fyn} / \mathrm{Cd} 2 \mathrm{ap}$ mice. Consistent with a previous report (20), we did not detect any proteinuria in Synpo-deficient or Synpo-heterozygous animals over the year of observation (Figure 3A). In the presence of CD2AP haploinsufficiency, however, $58 \%$ of Synpo ${ }^{-/-}$and $29 \%$ of Synpo ${ }^{+/-}$mice developed proteinuria. $(P<0.00001$ and $P<0.006$ respectively) (Figure $3 \mathrm{~A})$. The average age of onset of proteinuria was $7-8$ months and was not significantly different between $\mathrm{Synpo}^{+/-} \mathrm{Cd} 2 a \mathrm{p}^{+/-}$and Synpo-/-Cd2app $\mathrm{P}^{+/-}$mice (Figure 3A). Synpo $0^{+/-} \mathrm{Cd} 2 \mathrm{ap}^{+/-}$and Synpo$C d 2 a p^{+/-}$mice measured near the onset of proteinuria had serum BUN $(17.2 \pm 0.9 \mathrm{mg} / \mathrm{dl})$ and creatinine $(0.46 \pm 0.02 \mathrm{mg} / \mathrm{dl})$ levels $(n=27)$ that were similar to those of wild-type mice (129 background). Four mice were measured 2-3 months after onset of proteinuria, with elevated BUN $(50.4 \pm 16.5 \mathrm{mg} / \mathrm{dl})$ and creatinine levels $(0.7 \pm 0.13 \mathrm{mg} / \mathrm{dl})$. Proteinuria increased consistently from $90 \pm 6 \mathrm{mg} / \mathrm{dl}$ to $240 \pm 44 \mathrm{mg} / \mathrm{dl}$ when mice were followed for 2-3 months after onset of proteinuria $(n=6)$. As with the Cd2ap/Fyn mice, these data indicate a progression of disease over time with worsening severity of proteinuria, leading to an eventual decline in glomerular filtration and significant glomerulosclerotic changes.

\section{Figure 2}

Electron micrograph images from combined heterozygous mice between Cd2ap and Fyn. (A) Representative electron microscopic images showing normal foot process architecture in wild-type (left panel), Cd2ap ${ }^{+/}$(middle panel), and $\mathrm{Fyn}^{+/-}$(right panel) podocytes. (B) Electron micrographs from proteinuric $\mathrm{Fyn}^{-/}$mice revealed only some coarsening of the foot processes. (C and D) Fyn ${ }^{+-} \mathrm{Cd} 2 \mathrm{ap}^{+-}$mice (C) and $\mathrm{Fyn}^{-/-} \mathrm{Cd} 2 \mathrm{ap}^{+/-}$mice (D) exhibited foot process effacement in some but not all areas. Magnification, $\times 12,000$.

Light microscopic examination of Synpo+/- and Synpo-/kidneys did not reveal any obvious histological abnormalities (Figure 3B), but pathological changes were obvious in Synpo $0^{+/-} \mathrm{Cd} 2 \mathrm{ap}^{+/-}$and Synpo ${ }^{-/-} \mathrm{Cd} 2 \mathrm{ap}^{+/-}$kidneys (Figure 3B). As with the Fyn/CD2AP mice, these changes mainly involved hypercellular glomeruli with expanded mesangium in some but not all glomeruli. In addition, there were occasional glomeruli that were either completely or segmentally sclerosed (Figure 3B).

Electron microscopic examination of the kidneys from these mice showed no obvious changes in kidneys from Synpo ${ }^{+/-}$or Synpo-/- mice. In the presence of CD2AP haploinsufficiency, however, both Synpo $0^{+/-}$and Synpo-/- mice demonstrated sequential changes in the foot process architecture, consisting of coarsening and effacement of foot processes (Figure 4, B and C). We noted the presence of electron-dense deposits within glomerular basement membranes and mesangium (Figure 4B). Similar deposits and IgG staining have been seen in older (> 9 month old) Cd2ap heterozygous mice, as previously shown (13).

Electron microscopy and immunofluorescence staining for IgG

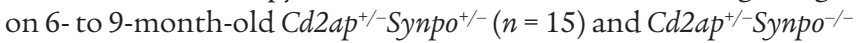
mice $(n=26)$ (data not shown) showed positive IgG staining in 13 out of 25 proteinuric mice and 8 out of 16 nonproteinuric mice. Electron-dense deposits were seen in 8 out of 23 proteinuric mice and 4 out of 16 nonproteinuric mice. Since the presence or absence of electron-dense deposits and IgG staining does not appear to correlate very well with proteinuria, we suspect that the presence of electron-dense deposits and positive IgG staining is related to the Cd2ap heterozygous background of the mice and not to the combined heterozygosity.

CD2AP haploinsufficiency combined with Neph1 heterozygosity. Neph1 is a transmembrane receptor closely related to nephrin, expressed in podocytes, brain, and smooth muscle of liver, lung, and heart (11). In the podocyte, Neph1 localizes to the slit diaphragm and is thought to function like nephrin in maintenance of the slit diaphragm $(22,23)$. Neph1-deficient animals, generated by retroviral insertion, are born with congenital nephrotic syndrome and usually die in the perinatal period (11).

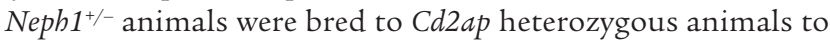
determine whether combined heterozygosity would contribute to a glomerular phenotype. Because of the mixed backgrounds of these animals, we compared only littermates, and the results

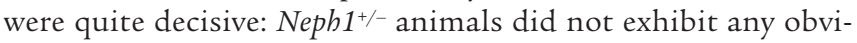
ous kidney abnormalities (data not shown). Zero of $16 \mathrm{Neph1^{+/- }}$ $C d 2 a p^{+/-}$mice developed proteinuria over the 12 -month observation period, and none of these mice exhibited any histologic abnormalities before 9 months of age (data not shown). Since bigenic heterozygosity of Neph1 and Cd2ap did not result in pro- 


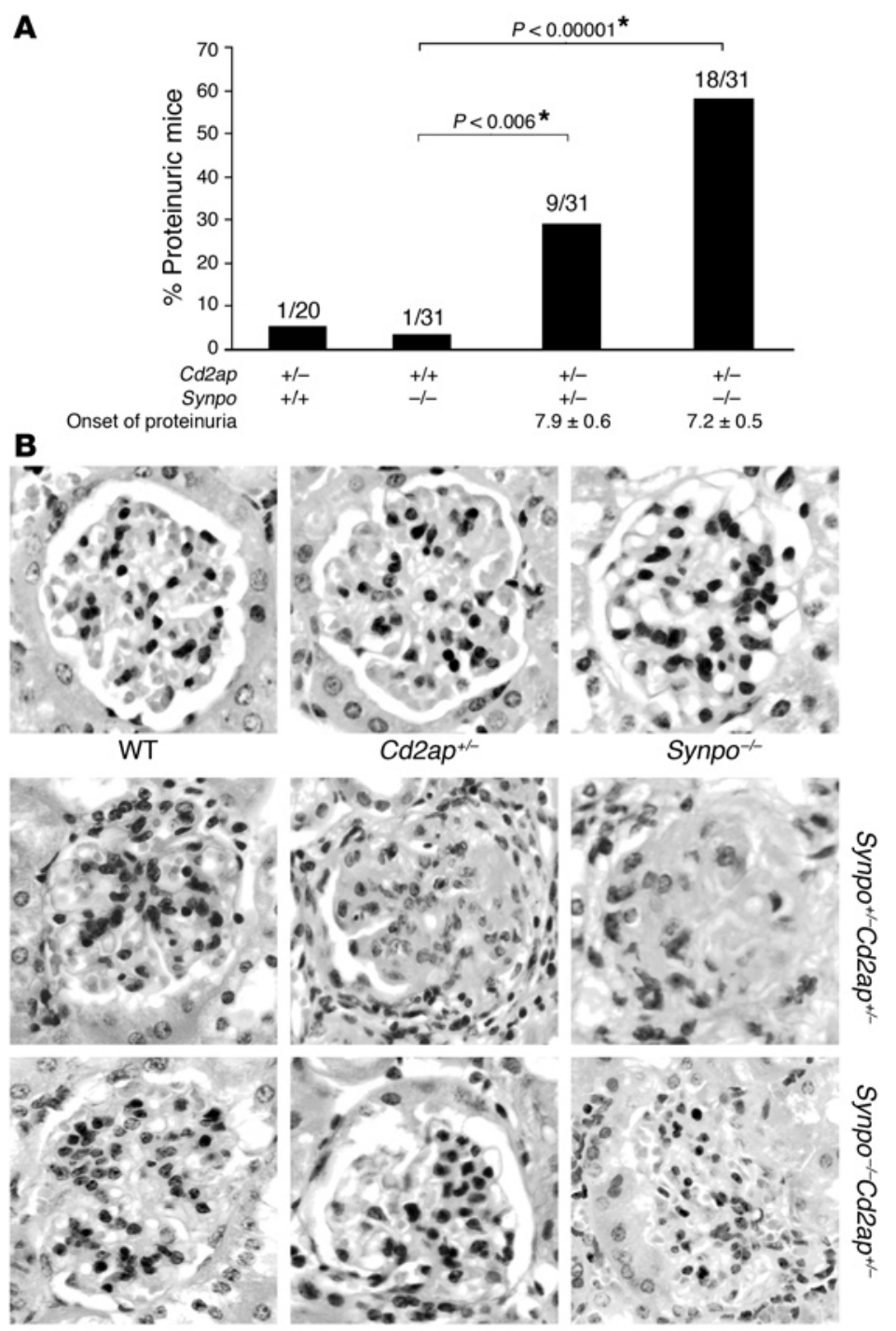

\section{Figure 3}

Bigenic Cd2ap and Synpo heterozygosity lead to proteinuria and FSGS. (A) Mice were screened monthly for proteinuria. The incidence of proteinuria is depicted as both percentages and absolute numbers for the indicated mouse genotypes followed for up to 12 months. The onset of proteinuria is shown below for each proteinuric genotype in months \pm SEM and did not differ among genotypes. *Statistical significance. (B) Sections of formalin-fixed kidney cortex from 6- to 9-monthold mice were stained with $\mathrm{H} \& \mathrm{E}$ and with PAS. In wild-type, Synpo ${ }^{-/}$, and $C D 2 A P^{+/-}$mice, nearly all glomeruli were normal (top panel). Representative glomeruli from Synpo ${ }^{+/-} \mathrm{Cd} 2 \mathrm{ap}^{+/-}$ mice (middle row) or Synpo ${ }^{-/-} \mathrm{Cd} 2 \mathrm{ap}^{+/-}$mice (bottom row) 2 months after the onset of proteinuria showed mesangial hypercellularity and segmental or global sclerosis in some but not all glomeruli. Magnification, $\times 400$.

with an antiserum to CD2AP. CD2AP was easily detected in Fyn but not in control antibody immunoprecipitates. This demonstrates for what we believe is the first time that endogenous CD2AP and Fyn form a constitutive complex (Figure 5A).

To test for an interaction between Synpo and CD2AP, immunoprecipitates were prepared from podocyte cell lysates using Synpo antibodies. Immunoblotting with antibodies to CD2AP showed the presence of CD2AP in Synpo lysates (Figure 5B). The converse was also true. Immunoprecipitates prepared with CD2AP antibodies and immunoblotted with antibodies to Synpo also demonstrated an interaction (Figure 5B).

To map the interacting domains, 2 mutated forms of $\mathrm{CD} 2 \mathrm{AP}$ were used. The structure of CD2AP is notable for 3 $\mathrm{SH} 3$ domains at the $\mathrm{N}$ terminus and multiple proline-rich sequences at the $\mathrm{C}$ terminus. Constructs lacking the $\mathrm{SH} 3$ domains or the proline-rich sequences were both tested for Synpo binding. While Synpo (Synpo-long) interacted with wild-type CD2AP and a mutated form of CD2AP lacking the proline sequences, there was no detectable interaction of Synpo (Synpo-long) with the mutated form of CD2AP teinuria, the combined heterozygous effect of Cd2ap with Fyn and Synpo appears to be specific.

Cd2ap heterozygosity does not alter blood pressure. Since CD2AP is widely expressed, including in blood vessels, myocardium, and renal collecting duct, we wanted to exclude the possibility that $C d 2$ ap heterozygosity accelerated glomerular disease by inducing hypertension. Mean arterial pressure (MAP) was measured using intraarterial pressure catheters in 4-month-old male wildtype and $C d 2 a p$ heterozygous animals $(n=7)$. Blood pressure was found to be normal and similar between $C d 2 a p$ heterozygous mice $(\mathrm{MAP}=70 \mathrm{mmHg})$ and littermate wild-type and wild-type controls ( $\mathrm{MAP}=71 \mathrm{mmHg})$.

CD2AP interacts with Fyn and Synpo, but not with Neph1. The above results showing genetic epistasis between CD2AP and Fyn and between CD2AP and Synpo suggested that these proteins may function in the same pathway. We were therefore interested to determine whether CD2AP could physically interact with either Synpo or Fyn. To test for interactions between CD2AP and Fyn, immunoprecipitates prepared from human kidney cell-line lysate (Figure 5A) or rat glomerular lysates (Figure 5A) using antibodies to Fyn (polyclonal antibody) were immunoblotted lacking the SH3 domains (Figure 6A). This suggests that the SH3 domains of CD2AP mediate the interaction with Synpo-long, presumably by binding to proline-rich sequences in Synpo.

To test whether the interaction between the SH3 domains of CD2AP and Synpo is direct, far Western blot experiments were performed. FLAG-tagged Synpo-long and FLAG-tagged GFP as control were blotted on a nitrocellulose membrane and subsequently incubated with biotinylated, recombinant CD2AP protein containing all $3 \mathrm{SH} 3$ domains. As shown in Figure 6B, the CD2AP SH3 domains specifically bound to Synpo-long, indicating a direct $\mathrm{SH} 3$ domain-mediated interaction between CD2AP and Synpo.

Recently, the existence of 3 Synpo isoforms, Synpo-long, Synposhort, and Synpo-T, has been described (20). We therefore tested whether CD2AP interacts with all isoforms of Synpo. Figure 6C shows that CD2AP can be coimmunoprecipitated with Synpolong, Synpo-short, and Synpo-T (Figure 6C).

Lastly, since CD2AP is known to interact with nephrin, we were interested to know whether CD2AP interacts with Neph1. Immunoprecipitates were prepared using lysates from cells coexpressing CD2AP with epitope-tagged Neph1 or epitope-tagged 
A

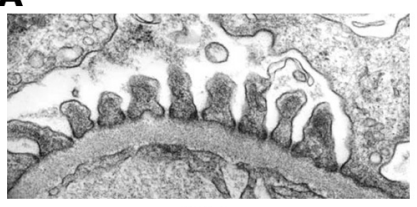

WT

\section{B}

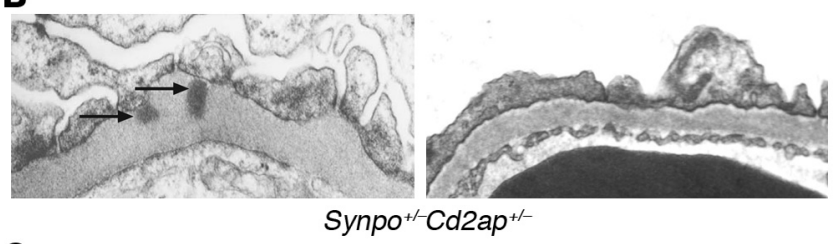

C

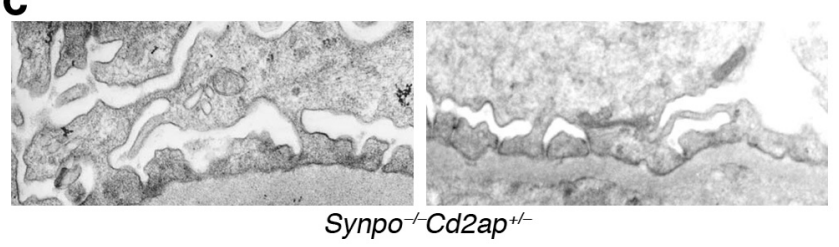

nephrin. While immunoblotting of CD2AP immunoprecipitates showed the presence of nephrin, no interaction between CD2AP and Neph1 was detected under the same conditions (Figure 6E).

\section{Discussion}

Here, we tested whether combined genetic heterozygosity might play a role in the etiology of FSGS. Given the limited number of proteins implicated in podocyte-specific functions, a simple approach was to directly test whether combined heterozygosity of podocyte-relevant genes might cooperate together to cause podocyte dysfunction and, subsequently, glomerular disease. CD2AP is a scaffold protein that interacts with a variety of proteins, including slit diaphragm, signaling, and cytoskeletal proteins (24). We speculated that mutations in Cd2ap might sensitize the podocyte to mutations in a wide variety of different genes. We found that heterozygosity of $C d 2 a p$ combined with heterozygosity of Synpo or Fyn led to a significant incidence of glomerular dysfunction as evidenced by proteinuria and pathological changes consistent with FSGS.

Recent progress has helped to illuminate the important role of the podocyte in the etiology of glomerular diseases like FSGS $(2,3)$. Mouse knockout models demonstrate that deficiencies of either podocyte-specific genes (encoding for podocin, ref. 8; nephrin, ref. 25; and Neph1, ref. 11) or more ubiquitously expressed genes (encoding for $\alpha$-actinin-4, ref. 9; CD2AP, ref. 12; and Fyn, ref. 15) can result in podocyte dysfunction leading to a severe congenital nephrotic syndrome. Genetic studies in humans suggest that less severe mutations in some of these genes may be responsible for a more variable pattern of disease that may be responsible for some cases of familial and idiopathic FSGS (26).

Podocin mutations were first described in an autosomal recessive form of FSGS that occurs with variable onset in children between 2 and 10 years of age (4). Over 40 different mutations in podocin have now been described (27). Most of the affected patients with autosomal recessive

\section{Figure 4}

Electron micrograph images from combined heterozygous mice between Cd2ap and Synpo. (A) Representative electron microscopic images showing normal foot process architecture in wild-type (left panel) or $\mathrm{Cd} 2 \mathrm{ap}^{+/-}$mice (right panel). (B) Electron micrographs from proteinuric Synpo ${ }^{+-} C D 2 A P^{+/-}$showed extensive foot process effacement as well as subepithelial deposits (arrows). (C) Electron micrographs from proteinuric Synpo ${ }^{-/-} \mathrm{CD} 2 \mathrm{AP}^{+/-}$showed findings similar to those shown in B, with foot process effacement. Magnification, $\times 12,000$.

FSGS have homozygous missense mutations, suggesting that the mutation causes a weak loss of function that is only able to cause disease when mutations of both alleles are present (27). Importantly, cases of increased disease susceptibility have been reported with mutations of only 1 podocin allele, suggesting involvement of another genetic locus $(4,26)$.

Mutations in the gene encoding $\alpha$-actinin- 4 are linked to an autosomal dominant form of FSGS in which the clinical presentation is also widely variable (5). In these patients, the disease has a late onset (first occurring in early adulthood) and is incompletely penetrant (5). Mice that are completely deficient for $\alpha$-actinin-4 develop a severe congenital nephrotic syndrome (9) while mice that are heterozygous for the dominant acting K228E allele do not display any overt disease (28). These observations strongly suggest that environmental as well as other genetic factors may play important roles in determining whether disease occurs when a single copy of the disease-causing allele is present.

Here, we focused on bigenic heterozygosity of $C d 2 a p$. We previously showed that mice that are heterozygous for Cd2ap develop pathological changes after 9 months of age but not proteinuria (13). Cd2ap heterozygous mice, however, have an increased sensitivity to nephrotoxic injury (13). Given the mild phenotype of $C d 2 a p$ heterozygous mice, we speculated that combined heterozygous mutations in Cd2ap and other associated proteins might increase susceptibility to podocyte dysfunction.

We first focused on combining Cd2ap heterozygosity with deficiency of the Src family tyrosine kinase Fyn. While $\mathrm{Fyn}^{+/-}$mice are
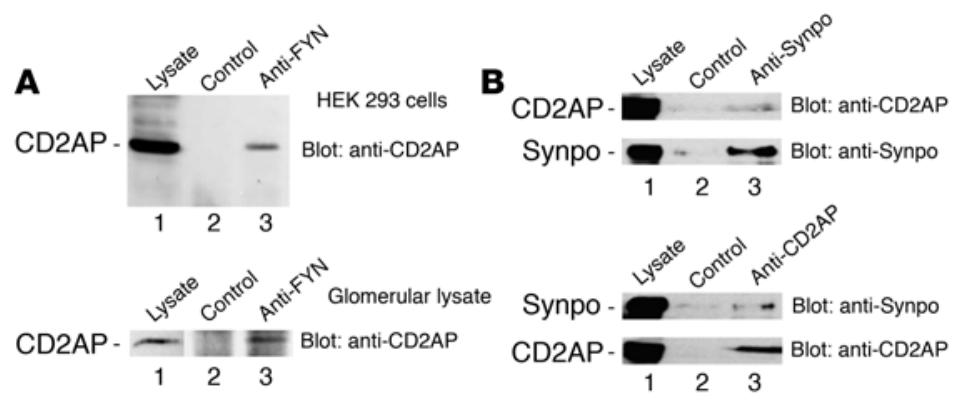

Figure 5

CD2AP forms an endogenous complex with Fyn and Synpo. (A) Endogenous CD2AP interacts with endogenous Fyn in cells (upper panel) and rat glomeruli (lower panel). Lysates were prepared from HEK 293 cells or isolated rat glomeruli, respectively (input lysate, $25 \mu \mathrm{l}$ out of $800 \mathrm{ml}$; lane 1). Immunoprecipitates were prepared with a control antibody (rabbit serum, lane 2 ) or with polyclonal antisera to Fyn (lane 3). Immunoblotting was performed with a polyclonal antibody to CD2AP. (B) Endogenous CD2AP interacts with endogenous Synpo in podocytes. Lysates were prepared from differentiated podocytes. Immunoprecipitates were prepared with a control antibody and anti-Synpo (upper panel) or anti-CD2AP (lower panel) antibodies and immunoblotted with anti-Synpo or anti-CD2AP. 

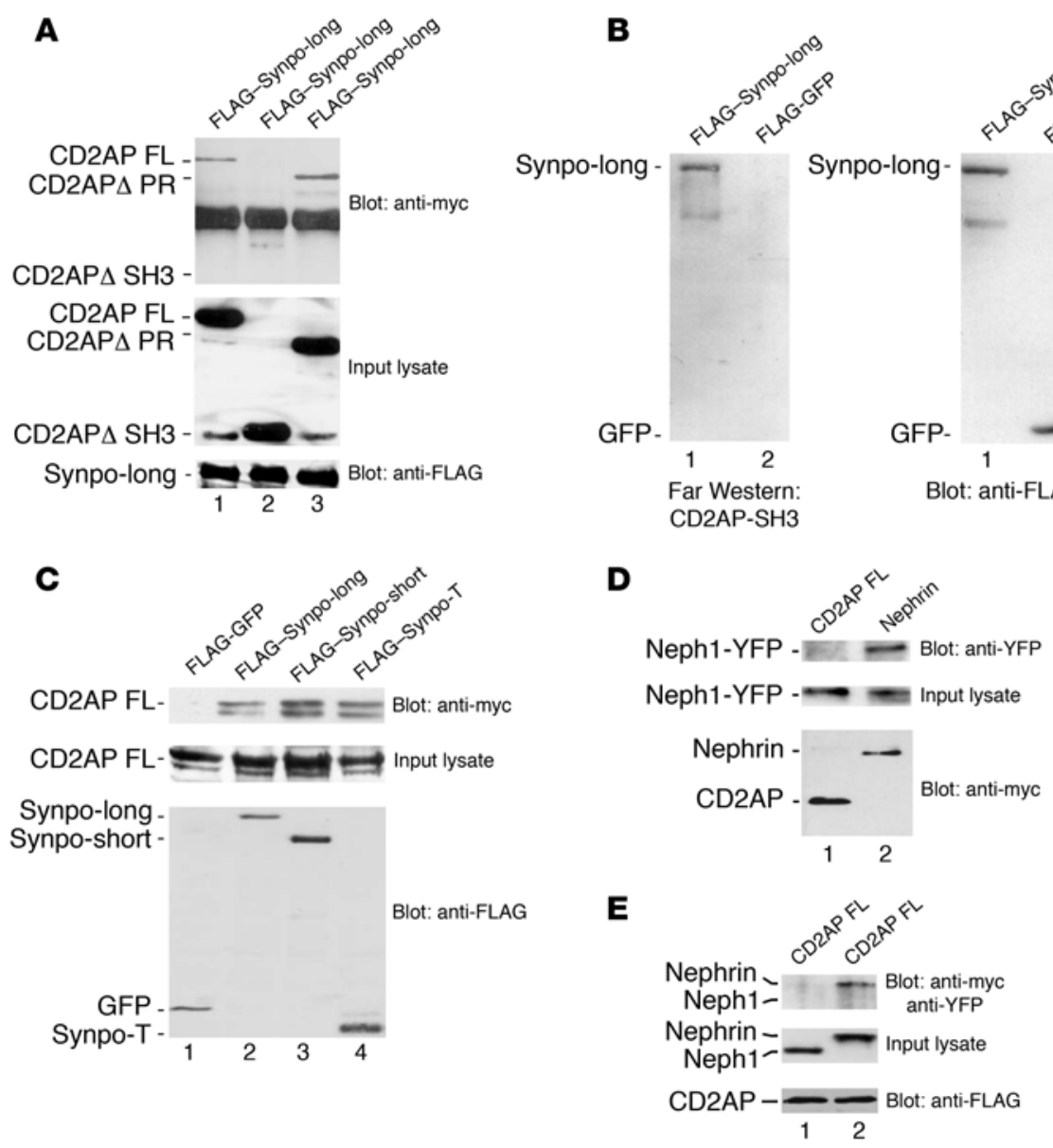

\section{Figure 6}

CD2AP SH3 domains interact directly with Synpo, but CD2AP does not interact with Neph1. (A) FLAG-tagged Synpo (Synpo-long) was coexpressed with myc-tagged proteins, including fulllength CD2AP (lane 1, CD2AP FL), CD2AP lacking the SH3 domains (lane 2, $\triangle \mathrm{SH} 3$ domains), and CD2AP lacking the proline-rich domains (lane 3, $\triangle \mathrm{PR}$ domains). Anti-FLAG immunoprecipitates were immunoblotted with an anti-myc antibody (upper panel). The expression of wild-type and mutated CD2AP proteins (input lysate; anti-myc) and Synpo is shown in the middle and lower panels, respectively. (B) FLAG-tagged Synpo (lane 1, FLAG-Synpo-long) and FLAG-tagged GFP (lane 2) were overexpressed, and anti-FLAG immunoprecipitates were blotted with recombinant, biotinylated GST-CD2AP fusion protein containing the $3 \mathrm{SH} 3$ domains (left panel) or immunoblotted with anti-FLAG antibody (right panel). (C) FLAG-tagged GFP (lane 1, control), FLAG-tagged Synpo-long (lane 2), FLAG-tagged Synpo-short (lane 3), and FLAG-tagged Synpo-T (lane 4) were coexpressed with myc-tagged full-length CD2AP. Immunoprecipitates were prepared with an antiFLAG antibody and immunoblotted with an anti-myc antibody (upper panel). (D) Neph1-yellow fluorescent protein (Neph1-YFP) was coexpressed with myc-tagged full-length CD2AP (lane 1) or myc-tagged nephrin (lane 2). Anti-myc immunoprecipitates were immunoblotted with anti-YFP antibody (upper panel). The expression of Neph1-YFP input lysate and myc-tagged nephrin or CD2AP controls is shown in the middle and lower panels, respectively. (E) Neph1-YFP (lane 1) and myc-nephrin (lane 2) were coexpressed with full-length FLAG-tagged CD2AP. Immunoprecipitates of FLAG-CD2AP were immunoblotted with anti-myc and anti-YFP antibodies. Neph1-YFP or mycnephrin input lysates (anti-myc, anti-YFP) and CD2AP expression are demonstrated in the middle and lower panels, respectively.

phenotypically and histologically normal, 2 groups have demonstrated that podocyte foot processes are abnormal in $\mathrm{Fyn}^{-/-}$mice $(14,15)$. The occurrence of proteinuria in these mice, however, is controversial, with DeFranco's group (15) reporting proteinuria in most mice by 6 months of age and Holzman's group (14) not reporting any proteinuria in these mice. In our study, we found about $31 \%$ of $\mathrm{Fyn}^{-/-}$mice develop proteinuria by 12 months of age. This value, which is intermediate between the results of the 2 previous studies, suggests that other factors may be involved in the initiation and/or severity of proteinuria. However, the combination of CD2AP haploinsufficiency with Fyn deficiency clearly accentuated disease, as $100 \%$ of these animals developed proteinuria. A gene dosage effect was also apparent, since mice that were heterozygous for $C d 2 a p$ and null for Fyn developed proteinuria at a rate that was almost double the rate of combined $\mathrm{Cd} 2 \mathrm{ap}^{+/-}$and $\mathrm{Fyn}^{+/-}$mice. Lastly, it should be noted that bigenic heterozygosity resulted in pathological changes that were not present in the $\mathrm{Fyn}^{+/-}$or $\mathrm{CD} 2 \mathrm{AP}^{+/-}$mice.

Our experiments with the Synpodeficient mice implicate, for what we believe is the first time, a role for Synpo in the pathogenesis of FSGS. Synpo is an actin-bundling protein that is specifically expressed in podocytes and in certain neurons in the brain (19). Synpo knockout mice exhibit neuronal defects but do not exhibit any spontaneous kidney abnormality $(20,21)$. Recently, the discovery of multiple Synpo isoforms and the fact that the shortest Synpo isoform (Synpo-T) is still expressed in the Synpo knockout suggest that this isoform might be compensating for the loss of the larger 2 isoforms in podocytes (20). The in vivo functionality of Synpo-T is not entirely clear, but in any case, our work establishes that Synpo$\mathrm{T}$ is unable to fully compensate in the presence of CD2AP haploinsufficiency.

It should be emphasized that the relatively mild pathological changes seen here are partially related to the way the experiment was designed. We used proteinuria as the end-point and sacrificed mice as soon as proteinuria was confirmed. Since we screened mice only monthly, the variability of proteinuria and pathological changes may have been due to variable length of time proteinuria was present. When a few proteinuric animals were followed for several months, the proteinuria and pathological changes progressed rapidly, leading to renal dysfunction and a significant level of glomerulosclerosis.

These studies demonstrating genetic epistasis among CD2AP, Fyn, and Synpo imply that these proteins might interact with each other. We showed, for what we believe is the first time, endogenous associations of CD2AP with Synpo and Fyn. The association of Fyn 
with CD2AP is probably mediated by the SH3 domain of Fyn binding to proline sequences in $\mathrm{CD} 2 \mathrm{AP}$, as a previous report showed that the SH3 domains of Fyn, Src, and Yes could bind to CD2AP in vitro (29). Our study also demonstrated that Synpo and CD2AP form a protein complex. Mapping studies revealed that CD2AP and Synpo can directly interact and that this interaction is mediated by the SH3 domains of CD2AP, presumably by binding to proline-rich segments of Synpo. The fact that CD2AP binds to the nonoverlapping isoforms Synpo-short and Synpo-T suggests that there are at least 2 different proline-rich segments that can bind to CD2AP.

These data suggest that CD2AP, Fyn, and Synpo act in the same genetic pathway to maintain podocyte integrity. While the specific pathway is unclear, clues have emerged from the wide variety of proteins that are known to associate with CD2AP. For instance, $\mathrm{CD} 2 \mathrm{AP}$ has been shown to interact with the slit diaphragm proteins podocin (30) and nephrin (31). A putative role for CD2AP in linking the slit diaphragm to the cortical actin cytoskeleton is suggested by associations of CD2AP with actin-capping protein $(32)$, cortactin $(33,34)$, and the GTPase-activating protein for ADP-ribosylation factor (ARF) family G proteins, ASAP1 (35). $\mathrm{CD} 2 \mathrm{AP}$ is also implicated in intracellular trafficking by its known interactions with proteins, such as synaptojanin, Rab4, and AP-2 $(36,37)$. A role for CD2AP in mediating ubiquitination and degradation in the lysosome is indicated by its known association with the ubiquitin ligase $\mathrm{Cbl}(38,39)$.

In this study, we identified 2 new interacting partners for CD2AP, Fyn, and Synpo. As Fyn is known to tyrosine phosphorylate nephrin, it is exciting to speculate that the slit diaphragm is a type of signaling receptor and that $\mathrm{CD} 2 \mathrm{AP}$ functions to orchestrate such complex processes as endocytosis and turnover of slit diaphragm components, establishments of cell polarity, and antiapoptotic signaling. The interaction between CD2AP and Synpo reinforces the idea that $\mathrm{CD} 2 \mathrm{AP}$ plays a central role in regulating the actin cytoskeleton. We speculate that combined heterozygosity of CD2AP and many other proteins in the slit diaphragm signalosome may contribute to susceptibility to FSGS.

It is somewhat surprising that there are only a few published models where bigenic heterozygosity has been shown to directly lead to disease. These include congenital nonsyndromic hearing impairment (40), human insulin resistance $(41,42)$, inherited disorders of fatty acid $\beta$-oxidation (43), and polycystic kidney disease (44). To our knowledge, this is the first description of combined heterozygosity causing any kind of glomerular disease. Koziell et al. reported a "triallelic" phenotype of 4 patients with homozygous mutations in either Nphs1 (nephrin) or Nphs2 (podocin), with an additional mutation in 1 allele of either Nphs 1 (nephrin) or Nphs2 (podocin) (45). Genetic epistasis between nephrin and podocin is suggested by the combined effects of the 3 mutations (45). It is not clear, however, how the presence of the heterozygous allele influences the phenotype or the course of disease. But this reinforces the idea that the presence of additional mutations may play a role in susceptibility to FSGS.

The initial therapy for most cases of FSGS involves steroids or other potent immunosuppressants; however, the FSGS seen in established disease-causing mutations has typically been poorly responsive to steroids (46). Only a small fraction of the cases of steroid-resistant FSGS or familial FSGS have identified mutations (2). Thus, many of the remainder are likely to have some combinations of heterozygous mutations, as demonstrated in our models. The identification of such genes and alleles involved in familial FSGS will therefore aid the clinician in choosing which patients are likely to benefit from potentially toxic immunosuppressants. The future focus of glomerular diseases, such as FSGS, should expand from the evaluation of individual genes to the examination of the impact of many genes in combination. The use of combined mutational mouse models may allow us to identify genes that may act in concert with other genetic mutations to increase disease susceptibility.

\section{Methods}

Materials and reagents. The immortalized podocyte cell line has been described in detail (47). Synpo cDNA constructs (20), CD2AP- and nephrin-cDNA constructs (31) have been published previously. Antibodies to Synpo (20), to CD2AP (31), and to Fyn (48) have been described. Anti-Myc Tag (clone 4A6; Upstate USA Inc.), anti-FLAG M2-agarose (Sigma-Aldrich), monoclonal anti-GFP antibody (JL-8, BD Biosciences), protein G sepharose (Sigma-Aldrich), and Sulfo-NHS-LC-Biotin (Pierce Biotechnology) were purchased as indicated.

Mice breeding. CD2AP-deficient mice were maintained in our colony as heterozygotes and genotyped as previously described (12). Cd2a $\mathrm{p}^{+/-}$mice were backcrossed (>10 generations) to a C57BL/6J background or maintained on a pure $129 \mathrm{~Sv}$ background.

Fyn-deficient mice were obtained from Jackson Laboratory, maintained on a C57BL/6J background in our laboratory, and genotyped per their published protocol. Synpo-deficient mice on a 129/Ola background (20) were genotyped as previously described (21). Neph1-deficient mice were obtained from Lexicon Genetics Inc. on a mixed 129/C57BL/6J-Tyrc-brd background and genotyped as previously published (11).

Synpo/CD2AP and Fyn/CD2AP colonies were maintained on a pure 129 or C57BL/6J background, respectively. For all experiments, only littermates derived from the intercrossing of the 2 gene-deficient lines were used. In the case of the Neph1 and CD2AP intercrossing, we used colonies with a mixed C57BL/6J and 129Sv background. However, again we compared only littermates.

All animal studies reported here were approved by the Washington University Animal Studies Committee.

Urine and serum protein and creatinine. Urine was collected monthly and tested for proteinuria using SDS-PAGE and known quantities of BSA as controls. An albumin concentration of $30 \mathrm{mg} / \mathrm{dl}$ was considered positive. Results correlated with measurements of urine protein performed using a Roche Cobas Mira Plus analyzer (Roche Diagnostics Corp.).

Histology. Mice were sacrificed within a month of scoring positive for proteinuria. After dissection, kidneys were rinsed in phosphate-buffered saline, fixed overnight in formalin, and submitted to the Histopathology Laboratory at Washington University Medical School for paraffin embedding, sectioning, and staining with PAS and H\&E

Electron microscopy. After dissection, renal cortex was fixed in $2.5 \%$ glutaraldehyde in $0.1 \mathrm{M} \mathrm{Na}$ cacodylate, stained in $\mathrm{OsO}_{4}$, dehydrated in ethanol, embedded in PolyBed 812 (Polysciences Inc.), sectioned on an ultramicrotom (Ultra Cut, Reichert-Jung), poststained in uranyl acetate and lead citrate, then viewed on a Zeiss 902 Electron Microscope in the Cell Biology Department of Washington University.

Blood pressure measurements. Mice were anesthetized with a $1.5 \%$ isoflurane/oxygen mixture administered with a vaporizer (Ohmeda Isotec 3, BOC Health Care). As previously described (49), we recorded heart rate and MAP by catheterizing the right common carotid artery with a Millar 1.4 French blood pressure probe and transducer (Millar Instruments Inc.) coupled to a PowerLab/4SP data-acquisition system (ADInstruments) and passing the probe into the ascending aorta.

Coimmunoprecipitation. HEK 293 T cells were transiently transfected with Lipofectamine (Invitrogen Corp.). After incubation for 24 hours, cells were 
washed twice and lysed in a $1 \%$ Triton X-100 lysis buffer. To test the coimmunoprecipitation of nephrin and Neph1 with CD2AP, we used a lysis buffer containing $1 \%$ digitonin (31). After centrifugation $(15,000 \mathrm{~g}, 15 \mathrm{~min}$, $4^{\circ} \mathrm{C}$ ), cell lysates containing equal amounts of total protein were incubated for 1 hour at $4^{\circ} \mathrm{C}$ with the appropriate antibody followed by incubation with $40 \mu \mathrm{l}$ of protein $\mathrm{G}$ sepharose beads for approximately 1 hour. The beads were washed extensively with lysis buffer, and bound proteins were resolved by $10 \%$ SDS-PAGE, transferred to nitrocellulose membranes, and immunoblotted with specific antibodies. For coimmunoprecipitation of endogenous proteins, lysates were prepared from $293 \mathrm{~T}$ cells, differentiated mouse podocytes, or rat glomerular lysates. After centrifugation $(15,000 \mathrm{~g}$, 15 minutes, $\left.4^{\circ} \mathrm{C}\right)$ and ultracentrifugation $\left(100,000 \mathrm{~g}, 30\right.$ minutes, $\left.4^{\circ} \mathrm{C}\right)$, cell lysates containing equal amounts of total protein were precleared with protein $\mathrm{G}$ sepharose and then incubated for 1 hour at $4^{\circ} \mathrm{C}$ with the indicated antiserum, followed by incubation with $40 \mu$ l of protein $\mathrm{G}$ sepharose beads for approximately 1 hour. The beads were washed extensively with lysis buffer, and bound proteins were resolved by $10 \%$ SDS-PAGE.

Far Western blot. Immunoprecipitates of FLAG-tagged Synpo-long and FLAG-tagged GFP as control were run on SDS-PAGE and then transferred onto a nitrocellulose membrane. Blots were blocked in PBS-Tween 20 containing 5\% BSA and then incubated with $100 \mu \mathrm{g} / \mathrm{ml}$ of biotinylated $\mathrm{CD} 2 \mathrm{AP} \mathrm{SH} 3$ domains containing GST fusion protein in the blocking buffer at $4{ }^{\circ} \mathrm{C}$. The blots were probed with HRP-conjugated streptavidin and then subjected to enhanced chemiluminescence.

1. Kitiyakara, C., Kopp, J.B., and Eggers, P. 2003. Trends in the epidemiology of focal segmental glomerulosclerosis. Semin. Nephrol. 23:172-182.

2. Pollak, M.R. 2003. The genetic basis of FSGS and steroid-resistant nephrosis. Semin. Nephrol. 23:141-146.

3. Fogo, A.B. 2003. Animal models of FSGS: lessons for pathogenesis and treatment. Semin. Nephrol. 23:161-171.

4. Boute, N., et al. 2000. NPHS2, encoding the glomerular protein podocin, is mutated in autosomal recessive steroid-resistant nephrotic syndrome. Nat. Genet. 24:349-354.

5. Kaplan, J.M., et al. 2000. Mutations in ACTN4, encoding alpha-actinin-4, cause familial focal segmental glomerulosclerosis. Nat. Genet. 24:251-256.

6. Winn, M.P., et al. 2005. A mutation in the TRPC6 cation channel causes familial focal segmental glomerulosclerosis. Science. 308:1801-1804.

7. Reiser, J., et al. 2005. TRPC6 is a glomerular slit diaphragm-associated channel required for normal renal function. Nat. Genet. 37:739-744.

8. Roselli, S., et al. 2004. Early glomerular filtration defect and severe renal disease in podocin-deficient mice. Mol. Cell. Biol. 24:550-560.

9. Kos, C.H., et al. 2003. Mice deficient in $\alpha$-actinin4 have severe glomerular disease. J. Clin. Invest. 111:1683-1690. doi:10.1172/JCI200317988.

10. Kestila, M., et al. 1998. Positionally cloned gene for a novel glomerular protein--nephrin--is mutated in congenital nephrotic syndrome. Mol. Cell. 1:575-582.

11. Donoviel, D.B., et al. 2001. Proteinuria and perinatal lethality in mice lacking NEPH1, a novel protein with homology to NEPHRIN. Mol. Cell. Biol. 21:4829-4836.

12. Shih, N.Y., et al. 1999. Congenital nephrotic syndrome in mice lacking CD2-associated protein. Science. 286:312-315.

13. Kim, J.M., et al. 2003. CD2-associated protein haploinsufficiency is linked to glomerular disease susceptibility. Science. 300:1298-1300.

14. Verma, R., et al. 2003. Fyn binds to and phosphorylates the kidney slit diaphragm component nephrin. J. Biol. Chem. 278:20716-20723.

15. Yu, C.C., Yen, T.S., Lowell, C.A., and DeFranco, A.L.
2001. Lupus-like kidney disease in mice deficient in the Src family tyrosine kinases Lyn and Fyn. Curr. Biol. 11:34-38.

16. Gurley, S.B., et al. 2006. Impact of genetic background on nephropathy in diabetic mice. Am. J. Physiol. Renal Physiol. 290:F214-F222.

17. Qi, Z., et al. 2005. Characterization of susceptibility of inbred mouse strains to diabetic nephropathy. Diabetes. 54:2628-2637.

18. Hartner, A., et al. 2004. Characterization of the renal phenotype in a mouse model of Marfan syndrome. Virchows Arch. 445:382-388.

19. Mundel, P., et al. 1997. Synaptopodin: an actinassociated protein in telencephalic dendrites and renal podocytes. J. Cell Biol. 139:193-204.

20. Asanuma, K., et al. 2005. Synaptopodin regulates the actin-bundling activity of $\alpha$-actinin in an isoform-specific manner. J. Clin. Invest. 115:1188-1198. doi:10.1172/JCI200523371.

21. Deller, T., et al. 2003. Synaptopodin-deficient mice lack a spine apparatus and show deficits in synaptic plasticity. Proc. Natl. Acad. Sci. U. S. A. 100:10494-10499.

22. Barletta, G.M., Kovari, I.A., Verma, R.K., Kerjaschki, D., and Holzman, L.B. 2003. Nephrin and Neph1 co-localize at the podocyte foot process intercellular junction and form cis hetero-oligomers. J. Biol. Chem. 278:19266-19271.

23. Liu, G., et al. 2003. Neph1 and nephrin interaction in the slit diaphragm is an important determinant of glomerular permeability. J. Clin. Invest. 112:209-221. doi:10.1172/JCI200318242.

24. Huber, T.B., and Benzing, T. 2005. The slit diaphragm: a signaling platform to regulate podocyte function. Curr. Opin. Nephrol. Hypertens. 14:211-216.

25. Putaala, H., Soininen, R., Kilpelainen, P., Wartiovaara, J., and Tryggvason, K. 2001. The murine nephrin gene is specifically expressed in kidney, brain and pancreas: inactivation of the gene leads to massive proteinuria and neonatal death. Hum. Mol. Genet. 10:1-8.

26. Pereira, A.C., et al. 2004. NPHS2 R229Q functional variant is associated with microalbuminuria in the general population. Kidney Int 65:1026-1030.

27. Caridi, G., Perfumo, F., and Ghiggeri, G.M. 2005. NPHS2 (podocin) mutations in nephrotic syn- drome. Clinical spectrum and fine mechanisms. Pediatr. Res. 57:54R-61R.

28. Yao, J., et al. 2004. Alpha-actinin-4-mediated FSGS: an inherited kidney disease caused by an aggregated and rapidly degraded cytoskeletal protein. PLoS Biol. 2:e167.

29. Kirsch, K.H., Georgescu, M.M., Ishimaru, S., and Hanafusa, H. 1999. CMS: an adapter molecule involved in cytoskeletal rearrangements. Proc. Natl. Acad. Sci. U. S. A. 96:6211-6216.

30. Schwarz, K., et al. 2001. Podocin, a raft-associated component of the glomerular slit diaphragm, interacts with CD2AP and nephrin. J. Clin. Invest. 108:1621-1629. doi:10.1172/JCI200112849.

31. Shih, N.Y., et al. 2001. CD2AP localizes to the slit diaphragm and binds to nephrin via a novel C-terminal domain. Am. J. Pathol. 159:2303-2308.

32. Hutchings, N.J., Clarkson, N., Chalkley, R., Barclay, A.N., and Brown, M.H. 2003. Linking the $\mathrm{T}$ cell surface protein CD2 to the actin-capping protein CAPZ via CMS and CIN85. J. Biol. Chem. 278:22396-22403.

33. Lynch, D.K., et al. 2003. A cortactin-CD2-associated protein (CD2AP) complex provides a novel link between epidermal growth factor receptor endocytosis and the actin cytoskeleton. J. Biol. Chem. 278:21805-21813.

34. Welsch, T., Endlich, N., Kriz, W., and Endlich, K. 2001. CD2AP and p130Cas localize to different F-actin structures in podocytes. Am. J. Physiol. Renal Physiol. 281:F769-F777.

35. Liu, Y., Yerushalmi, G.M., Grigera, P.R., and Parsons, J.T. 2005. Mislocalization or reduced expression of Arf GTPase-activating protein ASAP1 inhibits cell spreading and migration by influencing Arf1 GTPase cycling. J. Biol. Chem. 280:8884-8892.

36. Cormont, M., et al. 2003. CD2AP/CMS regulates endosome morphology and traffic to the degradative pathway through its interaction with Rab4 and c-Cbl. Traffic. 4:97-112.

37. Brett, T.J., Traub, L.M., and Fremont, D.H. 2002. Accessory protein recruitment motifs in clathrinmediated endocytosis. Structure. 10:797-809.

38. Soubeyran, P., Kowanetz, K., Szymkiewicz, I., Langdon, W.Y., and Dikic, I. 2002. Cbl-CIN85endophilin complex mediates ligand-induced 
downregulation of EGF receptors. Nature. 416:183-187.

39. Dikic, I. 2002. CIN85/CMS family of adaptor molecules. FEBS Lett. 529:110-115.

40. Zheng, Q.Y., et al. 2005. Digenic inheritance of deafness caused by mutations in genes encoding cadherin 23 and protocadherin 15 in mice and humans. Hum. Mol. Genet. 14:103-111.

41. Kido, Y., et al. 2000. Tissue-specific insulin resistance in mice with mutations in the insulin receptor, IRS-1, and IRS-2. J. Clin. Invest. 105:199-205.

42. Savage, D.B., et al. 2002. Digenic inheritance of severe insulin resistance in a human pedigree. Nat. Genet. 31:379-384.
43. Schuler, A.M., et al. 2005. Synergistic heterozygosity in mice with inherited enzyme deficiencies of mitochondrial fatty acid beta-oxidation. Mol. Genet. Metab. 85:7-11.

44. Wu, G., et al. 2002. Trans-heterozygous Pkd1 and $\mathrm{Pkd} 2$ mutations modify expression of polycystic kidney disease. Hum. Mol. Genet. 11:1845-1854.

45. Koziell, A., et al. 2002. Genotype/phenotype correlations of NPHS1 and NPHS2 mutations in nephrotic syndrome advocate a functional interrelationship in glomerular filtration. Hum. Mol. Genet. 11:379-388.

46. Meyrier, A. 2005. Treatment of focal segmental glomerulosclerosis. Expert Opin. Pharmacother.
6:1539-1549

47. Mundel, P., et al. 1997. Rearrangements of the cytoskeleton and cell contacts induce process formation during differentiation of conditionally immortalized mouse podocyte cell lines. Exp. Cell Res. 236:248-258.

48. Gauen, L.K., et al. 1994. Interactions of p59fyn and ZAP-70 with T-cell receptor activation motifs: defining the nature of a signalling motif. Mol. Cell. Biol. 14:3729-3741.

49. Heximer, S.P., et al. 2003. Hypertension and prolonged vasoconstrictor signaling in RGS2-deficient mice. J. Clin. Invest. 111:445-452. doi:10.1172/ JCI200315598. 